\title{
Basic Model of Investigation Regularities of Fatigue Aging of Engineering Devices' Elements in Aerospace Industry
}

\author{
Alexander Chursin ${ }^{1}$, Alexander Semenov ${ }^{1,2,{ }^{*}}$ and Anna Ostrovskaya ${ }^{1}$ \\ ${ }^{1}$ People's Friendship University of Russia, ul. Miklukho-Maklaya 6, Moscow, 117198 Russia \\ ${ }^{2}$ Moscow Technological Institute, Leninsky prospect, 38a, 119334 Russia
}

\begin{abstract}
The article deals with the general approach to the problem of fatigue of materials and elements in aerospace industry. The mathematical model describing the effect is presented and recommended for practical use. For solving different problems with the fatigue of the materials we recommend the described model with the given approximation type
\end{abstract}

\section{Introduction}

Mechanical properties of materials in aerospace industry are determined mainly on the basis of standard testing of samples according to the Russian standard GOST 286065. The main experimental information of these tests includes various types of fatigue curves used to determine the extreme values considered as properties. According to the approach used in this article, these curves are classified as standard fatigue curves of metal. We will outline the basic methodology based on the principles in [1]. The importance of new methods and innovations in aerospace industry is described in [2].

\section{Model of investigation regularities of fatigue aging}

Figure 1 illustrates the fatigue curve from Russian standard GOST 2860-65, which recommends to consider the horizontal plateau as an upper limit of complete fatigue strength (or $\sigma-1$ ). In Fig. 1:

$$
\sigma_{\max }=\sigma_{m}+\sigma_{a}
$$

where $\sigma_{\max }$ is the maximum stress of fatigue cycle; $\sigma_{m}$ is the static constituent of fatigue cycle, average cycle stress; $\sigma_{a}$ is the amplitude of cycle stress. This Standard does not present any special recommendations on approximation of the experimental data. In aerospace industry it is convenient to apply approximation by using polynomial equations for certain particular cases or statistic processing of the experimental data.

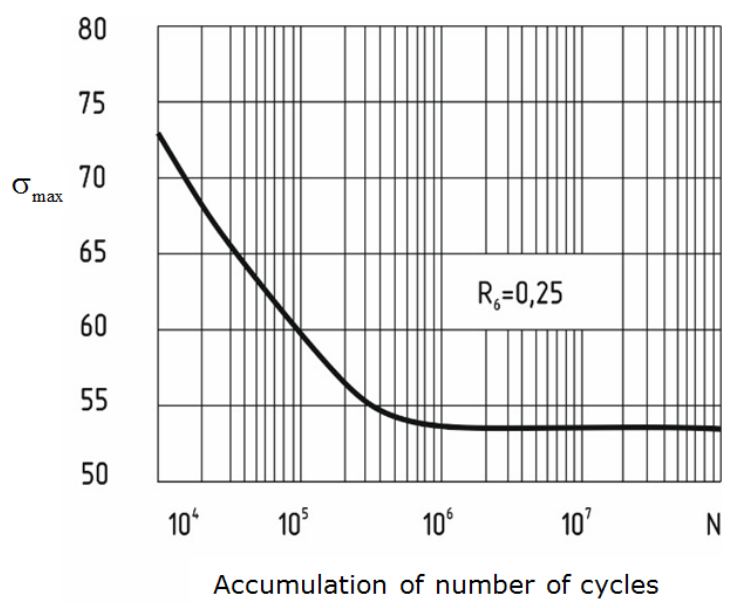

Fig. 1. Fatigue curve in coordinates $\sigma_{\max }, \ln N$

Approximation of experimental results using transcendental equations makes it possible to relate main constants of material with probabilistic parameters of their expected behaviour upon aging.

Fig. 2 illustrates fatigue curve from Fig. 1 in Cartesian coordinates. The values of $\sigma_{\max }$ for $N_{c}<104$ are extrapolated to $N_{c} \rightarrow 0$.

It is obvious that $\sigma_{\max }\left(N_{c}=0\right)$ is nothing else but ultimate strength upon static load. In this case a sample fails in the first load cycle. In this plot it is the second constant, asymptote, approached by the curve $\sigma_{\max }=f\left(N_{c}\right)$ at $N \rightarrow \infty$. Here and below the symbols $\sigma_{B}, \sigma_{R}$ and others are applied in common sense without relation to the specific type of the load or deformation (twisting, bend, compression, expanding and so on).

For practical cases we recommend the approximation to be performed by the method of selected points. Four points indicated by numbers are 
arbitrarily selected in the fatigue curve. Coordinates of these points are summarized in Tabl. $1\left(\sigma_{R}=52.5\right.$, $\left.\sigma_{B}=80\right)$.

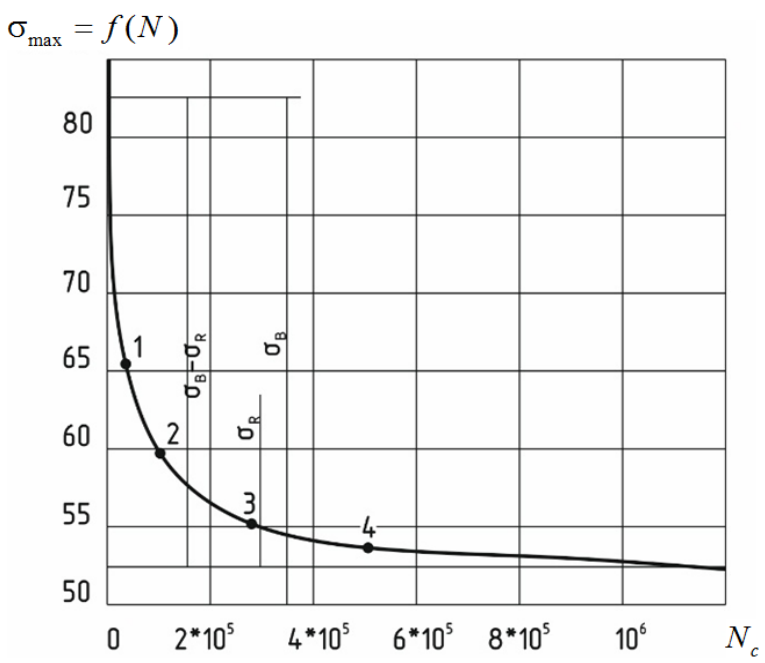

Fig. 2. Approximation of standard fatigue curve: $\sigma_{B}$ ultimate strength; $\sigma_{R}$ - fatigue strength.

Table 1. Initial data

\begin{tabular}{|c|c|c|c|c|c|}
\hline$^{\circ}{ }_{T T}$ & $N_{c i}$ & $\sigma_{\max }\left(N_{i}\right)$ & $^{\mathfrak{o}_{T T}}$ & $N_{c i}$ & $\begin{array}{c}\sigma_{\max } \\
\left(N_{i}\right)\end{array}$ \\
\hline 1 & 0.3 & 65 & 3 & 2.9 & 55 \\
& $10^{5}$ & & & $10^{5}$ & \\
\hline 2 & $110^{5}$ & 59 & 4 & $510^{5}$ & 53 \\
\hline
\end{tabular}

Standard fatigue curve is described as follows:

$$
\sigma_{\max }(N)-\sigma_{R}=\left(\sigma_{B}-\sigma_{R}\right) e^{-\mu N \alpha},
$$

A special case of (2) at $\alpha=1$ :

$$
\sigma_{\max }(N)-\sigma_{R}=\left(\sigma_{B}-\sigma_{R}\right) e^{-\lambda N}
$$

In (2), (3) $\mu$ and $\alpha$ are the deterministic analogues of the Weibull-Gnedenko distribution parameters; $\lambda$ is the intensity of decrease in strength (analogue of failure intensity). Transforming and applying the logarithm twice on (2) we obtain:

$$
\lg \left[-\ln \left(\frac{\sigma_{i}-\sigma_{R}}{\sigma_{B}-\sigma_{R}}\right)\right]=\lg \mu+\alpha \lg N
$$

Writing (4), for instance, for points 1 and 2 from Tabl. 1 and eliminating $\lg \mu$, we obtain:

For points 1 and 2:

$$
\alpha=\frac{1}{\lg \frac{0,3 \cdot 10^{5}}{1 \cdot 10^{5}}} \lg \left[\frac{\frac{65-52,5}{80-52,5}}{\frac{59-52,5}{80-52,5}}\right] .
$$

For points 3 and 4 :

$$
\alpha=\frac{1}{\lg \frac{10^{5}}{2.9 \cdot 10^{5}}} \lg \left[\frac{\ln \left(\frac{59-52.5}{80-52.5}\right)}{\ln \left(\frac{55-52.5}{80-52.5}\right)}\right]=0.4519
$$

The results of calculations are loosely approximated, thus, $\alpha=0.45$; and $\mu$ is determined by the following equation:

$$
\mu=-\frac{\ln \left(\frac{\sigma_{1}-\sigma_{R}}{\sigma_{B}-\sigma_{R}}\right)}{N_{i}^{\alpha}}
$$

For point 1 from Table 1:

$$
\mu_{1}=-\frac{\ln \left(\frac{65-52.5}{80-52.5}\right)}{\left(0.3 \cdot 10^{5}\right)^{0.45}}=8.62 \cdot 10^{-3}
$$

For point 2:

$$
\mu_{2}=-\frac{\ln \left(\frac{59-52.5}{80-52.5}\right)}{\left(10^{5}\right)^{0,45}}=8,69 \cdot 10^{-3}
$$

Calculation of the referential values by (5) gave nearly complete coincidence of the results.

\section{Conclusions}

Experimental application of (5) can be carried out by the following trends:

- selection of the load modes for a priori selected cumulative number for the load cycles NCyM. This procedure regulates selection of constrained load limit. Taking into account (1) and (2), the following equation can be derived:

$$
\sigma_{\alpha \max }(N)+\sigma_{\mathrm{m}}=\sigma_{\mathrm{R}}+\left(\sigma_{\mathrm{B}}-\sigma_{\mathrm{R}}\right) \mathrm{e}^{-\mu N \alpha},
$$

where $\sigma_{\text {amax }}(N)$ is the ultimate value of load amplitude; $\sigma_{m}$ is the static constituent, average cycle stress. Average cycle stress $\sigma_{m}$ is selected a priori, and ultimate value $\sigma_{\text {amax }}(N)$ is calculated;

- forecasting of expected probabilistic properties, taking into account that the parameters $\mu, \alpha, \lambda$ are identical to probabilistic dependences of the similar type.

So for solving different problems with the fatigue of the materials we recommend the described model with the given approximation type.

\section{References}

1. V. V. Efremov, V. A. Naumov, A. A. Chursin, Theory and practical issues of operability of elements of machines, devices and instruments, (Irkutsk University, Irkutsk, 1984) [In Rus]

2. A. A. Chursin, A. S. Semenov, A. V. Danilchankau, NPCS 19, 3 (2016) 